\title{
The Theory of Coherent Synchronized Reactions: Chemical Interference Logics
}

\author{
Tofik M. Nagiev
}

\begin{abstract}
Various types of possible interactions between reactions are discussed. Some of them are united by the general idea of chemical reaction interference. The ideas on conjugated reactions are broadened and the determinant formula is deduced; the coherence condition for chemical interference is formulated and associated phase shifts are determined. It is shown how interaction between reactions may be qualitatively and quantitatively assessed and kinetic analysis of complex reactions with under researched mechanisms may be performed with simultaneous consideration of the stationary concentration method. Using particular examples, interference of hydrogen peroxide dissociation and oxidation of substrates is considered. Therefore it is offered macrokinetic theory of coherent synchronized reactions.
\end{abstract}

Index Terms - Coherent, synchronize, interference, biomimetic.

\section{INTRODUCTION}

There are many types of interaction between reactions. Conjugated processes are the most demonstrative of reciprocal influence and interaction of two or more reactions.

The event when one reaction speeds up the proceeding of another reaction is shown up in non-chemical induction: e.g. via initiation or synthesis of a catalyst in a reaction for another reaction. The mutual influence of reactions synchronously proceeding in a system includes a much broader range of events than chemical induction suggests.

This chapter shows practically all kinds of possible reaction interactions, which part may be united in a general idea of interference of chemical reactions. The notion of interference includes mutual intensification or weakening of the reactions: for instance, the rate of primary reaction product formation decreases, whereas the rate of secondary, conjugated reaction product formation increases. Currently, the mutual influence of reactions synchronized in time and space will be taken for interfering chemical processes [1]-[3].

With this approach conjugated processes appear to be a particular case of interfering processes. As guided by the idea on chemical interference, one may define many kinds of interactions between reactions. Finally, the phenomenon may be formulated as follows: the event consisting of the fact that reactions synchronously proceeding in the chemical system are mutually intensified or weakened is called chemical interference, i.e. interaction (e.g. interfering) reactions must be coherent.

In modern chemistry the holistic approach to chemical studies is necessitated by solutions of general, fundamental

Manscript received November 25, 2014; revised January 9, 2015.

Tofik M. Nagiev is with the Research Center, Azerbaijan (e-mail: tnagiev@azeurotel.com). tasks. From this aspect, simultaneously proceeding chemical reactions represent the components of the entire chemical system, where, as the saying goes, 'all affects all'.

Synchronous processes represent the most demonstrativeand unique example of chemical reaction ensembles, arranged in time and space. Interest in synchronous chemical reactions is also so much keener, because in biological systems many processes are synchronous. This means that biochemical reactions are arranged and performed in systems with molecular and permolecular structures, which is the chemist's 'pipe dream'. Studies performed in recent decades have allowed the development of the interaction theory for synchronous chemical reactions at two levels - microscopic and macroscopic. Strictly speaking, parallel reactions may also be taken as synchronous reactions; although proceeding simultaneously in the reaction system, they are characterized by the absence of any interaction between them. However, such synchronous reactions are trivial and of no special interest for chemistry. It is of much more importance when they interact and, therefore, induce oscillations in yields of synchronous reaction products.

It should also be noted that processes are also synchronized via interactions of physical (primary) and chemical (secondary) processes. The microscopic physical process, which induces chemical reaction, is of a quantum type, e.g. highly active intermediate compounds (intermediates) for acceleration of the secondary reaction are formed by the physical (pulse) method. This microscopic coherence of synchronous processes has been described in detail by A.L. Buchachenko [4].

Macroscopic coherence of synchronous reactions is dually displayed, because at the macroscopic level chemical processes proceed in two zones - diffusional and kinetic.

Diffusional process and chemical reaction synchronization induces oscillations of reaction product yields. This common type of synchronous reactions in the literature is referred to as the Belousov-Zhabotinsky reaction.

Meanwhile, the majority of chemical and biochemical processes proceeds in the kinetic zone. Therefore, the synchronization of two chemical reactions or more is of special importance (Fig. 1 and Fig. 2).

As shown below, the observed intermodulation of synchronized chemical reaction yields is clearly displayed in the kinetic zone and represents a valid tool for voluntary manipulation of their rates.

For a fuller description, see the scheme shown in the next page reflecting all known types of synchronous reaction coherence.

The pulse induction and existence of the diffusional zone in a chemical reaction are conditions under which the primary 
physical process is synchronized with the secondary chemical reaction and, therefore, accelerates it.

Note that in the diffusional zone the chemical reaction rate is limited by the stage of initial substance supply (transport) to the place of chemical interactions, i.e. the chemical reaction rate is much higher than the rate of reagent diffusion to the active site.

On the other hand, the kinetic zone of chemical reaction proceeding is characterized by a much lower rate of chemical transformations than the rate of reagent transport to the active site, i.e. the diffusion rate of reagents is much higher than the chemical reaction rate. As follows from these canonical identifications, only the kinetic zone is associated with synchronization of purely chemical reactions, two at least. This circumstance is the main reason for significant differences in coherence conditions of various synchronous processes: pulse and diffusional synchronization methods permit the only one, unique chemical reaction.

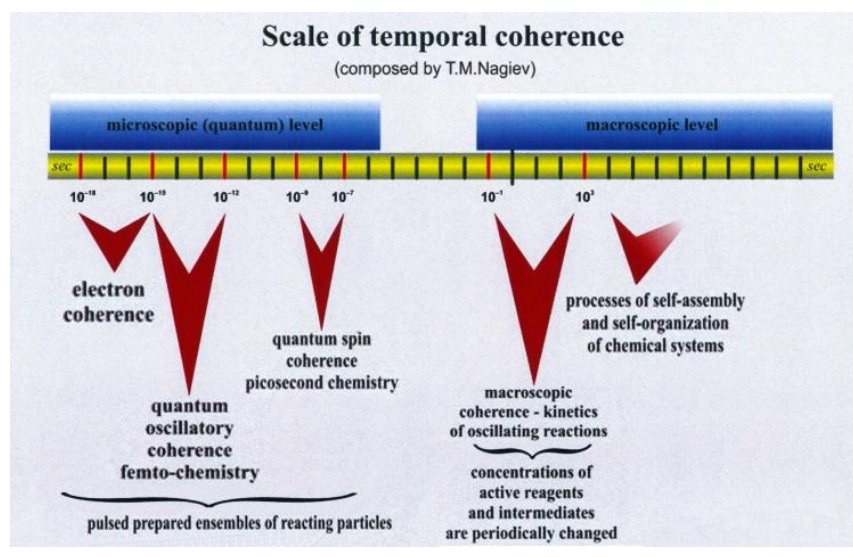

Fig. 1. Scale of temporal coherence.

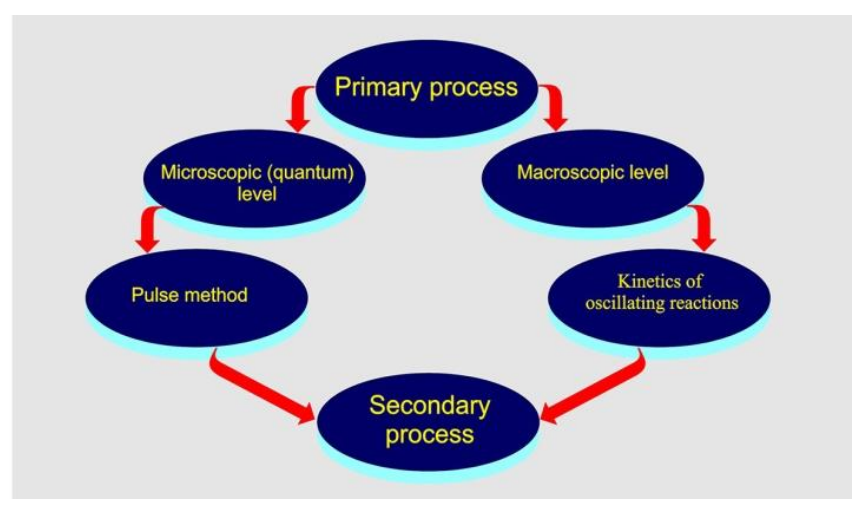

Fig. 2. Coherent synchronized processes.

Let us discuss this principal difference of two induction systems, physical and chemical, more comprehensively. In any chemical system, physical processes associated with diffusion and activation of reacting substances proceed simultaneously with chemical transformations of reagents. Therefore, as chemical reaction is induced by the associated physical process shaped as diffusion or activation represents the potential ability of any chemical system, whereas chemical induction consists of, at least, two coherently synchronized chemical reactions. As shown below, synchronization of kinetic curves for interfering chemical reaction product yields differs radically from these curves for physical influence on the secondary chemical reaction.

Despite origination of interrelated reactions in the early 1900s, the outlook for chemical interference is expected to be, if not extraordinary, but quite useful for interpretation of complex events and voluntary control of processes proceeding in chemical and biological systems.

Until recently, the expression 'chemical interference' was absent from chemical dictionaries, the terms 'complex reactions', 'chemical conjugation', 'induced reactions' and so on being most often used. In fact, the new term occurred in the late 1980s. It unites the majority of former terms concerning interaction and mutual influence of chemical reactions. This coining of this new term, unappreciated at first sight, is not merely to be attributed to an author who has thought up a new chemical term. It is actually of deeper significance. Most likely, it is associated with the complex situation appearing in chemistry and biology, when the fundamental tendency of modem biochemical investigations concludes in the development of a holistic approach to problems. Therefore, some chemical and biochemical processes may be barely or, sometimes not at all, understood without this approach.

In fact, chemical interference united scattered ideas about interactions and reciprocal influences and, therefore, formed the general fundamental basis for them.

Chemical interference reflecting the holistic approach to complex processes proceeding in chemical and biological systems, but not to their components as is commonly practiced, characterizes the phenomenon, which real sense justifies the introduction of a new term.

At the present time, the prospect of using chemical interference as the event becomes clearer still. It should be said that it may form the basis for creation of energetically favorable, highly selective and environmentally pure processes and, therefore, may affect economic aspects of applied developments.

With the aim of providing an in-depth and complete presentation of chemical interference, let us now discuss in detail the analysis of chemical interference as the phenomenon by revealing particular spheres of its application. We will also use the terminology usual for conjugated processes.

\section{INTER-REACTION INTERACTIONS TYPES}

As stated by N. A. Shilov, one of the most important objectives of conjugated reaction studies is ' ... determination how processes stipulating an event (of chemical induction) may be related to one class of conjugated reactions or another'. He has distinguished three types of conjugated processes related to a chemical system with two conjugated processes with three compounds participating in them. The total scheme of the conjugated process is as follows:

$$
\begin{gathered}
A+I n=R P(\text { primary reaction }) \\
A+A c c=R P(\text { secondary reaction })
\end{gathered}
$$

where $R P$ are reaction products.

The following typical features of conjugated reactions may be distinguished:

1) Energy-releasing reaction performs effective work for proceeding of another (heat-absorbing) reaction, i.e. free 
energy drop in the primary reaction fully covers the increase of free energy in the secondary reaction. It is also possible that spontaneous (secondary) reaction is accelerated, proceeding with the free energy decrease, but under current conditions its rate may be negligibly low (for instance, equal almost zero).

2) The principle of independent proceeding of elementary chemical reactions is not fulfilled.

3) The links between reactions are set via general intermediate compounds.

4) Conjugated reactions proceed in open systems only.

5) Conjugated reactions must be complex.

Principally, the reactions may be conjugated, when one of them slows another one down, and somewhat inhibits it. The mechanism of slowing down the secondary reaction may have different origins (e.g. if the target reaction is of the catalytic type, and intermediate products (IP) poisoning the catalyst are formed in the primary reaction). Another possible case is realized when IP of both reactions recombine or disproportionate (active sites are eliminated). Such negative effects of chemical reactions allow us to call this type of interaction the 'negative chemical induction' by analogy with the 'negative catalysis' notion [5]. Of interest is that in the case of negative chemical induction both reactions must be spontaneous. Otherwise, if one of the reactions proceeds with an increase in free energy, it is senseless to speak about the slowing down effect.

It should be noted that the acceleration of one reaction by another may also be manifested without chemical induction: e.g. via induction or synthesis of a catalyst for one reaction in another. The reciprocal influence of synchronously proceeding reactions in the system comprises a much wider range of events than does chemical induction.

Obviously, chemical induction is of great interest, because, on one hand, it allows the induction and acceleration of non-spontaneous reaction and, intrinsically, remains the unique method by which to affect such reactions (except for reactions proceeding under the influence of photochemical and ionizing radiation). On the other hand, chemical induction plays a significant role in biochemical processes. The literal translation from Latin term 'interference' is mutual (inter) collision (ferio), which shows the total situation.

As follows from the definition, interacting reactions may be called coherent ones.

Let us give the general definition of chemical induction, which reflects its specific features. At chemical induction, chemical reaction spontaneously proceeding in the system performs the effective work for another, non-spontaneous chemical reaction in the same system to proceed, which may not run without the first one.

In the current definition of chemical induction, we deliberately preserved the statement that duplicates the common one in order to focus readers' attention on the key statement of the definition. What is the principal difference between saying that the primary reaction 'induces' or 'performs effective work'? In principle, another reaction may be 'induced' by initiation, as well as by synthesis of a catalyst in the primary reaction. In these cases, the primary reaction does not make (thermodynamically) effective work for the secondary reaction to proceed. As defined, catalyst is unable to make effective work, and at initiation the target reaction is developed by a self-reaction pathway and needs a 'trigger mechanism' only at the initial stage of proceeding. In both cases, the target reaction is necessarily spontaneous. At chemical conjugation, the primary reaction continuously induces non-spontaneous process, making effective work during the reaction time. This is only possible during the change of the reaction mechanism or the target reaction type.

Let us now discuss in detail the analysis of chemical interference as the event giving the areas of its display in various forms with particular examples. Therefore, let us use the general terminology, common for characterization of conjugated processes.

In principle, connections and interactions between complex chemical reactions may be most varied. Fig. 3 gives the most significant of them, showing the layout of the most commonly known complex reactions: consecutive, parallel, consecutive-parallel, etc. At the moment, it should be noted that these schemes do not describe mechanisms of the processes at the level of elementary reactions. For consecutive reactions, IP are stable final substances of the first gross-stage, which are initial substances for the second stage. Fig. 3 also shows another type of intermediate compounds, which display extremely low stability (free radicals, labile complex compounds, etc.) and high reactivity in relation to the substrate.

To demonstrate this more clearly, reaction arrows for consecutive and parallel reactions, also displayed in Fig. 1, converge at a point from which, however, it does not follow that final products formed by all reactive pathways possess equal composition. Remember that these schemes most generally characterize complex reactions, and every direction shows formation of specific final products. As soon as these schemes obtain a particular shape, it becomes natural to indicate the corresponding final products for every reaction direction. Consecutive reactions are interconnected by synthesis of an IP at the first stage; thereafter, this product becomes the initial compound for the second stage. Intensification of the first complex reaction leads to expected intensification of another, dependent reaction. Among parallel reactions, the only reactions are reciprocally dependent, which contain even a single compound general for these reactions ( $A$ in the current scheme) in the compounding reaction.

As follows from Fig. 3, the change in concentration of substance $A$ caused by its general expenditure at different rates by the parallel reaction knots mentioned will affect their kinetics.

Therefore, parallel reactions implicitly affect proceeding kinetics of each other via the change of general reagent $A$ concentration. Since consecutive-parallel reactions represent a combination of those mentioned above, the type of interaction between individual complex reactions will be similarly performed (see Fig. 3).

In Fig. 3, conjugated processes are shown by the scheme clearly indicating its main features. In particular, it is clear that intermediate compounds formed in the primary reaction induce and speed up non-spontaneous secondary reactions between the actor and the acceptor. Hence, the type of 
secondary reaction is most often changed which, in a transformed shape, becomes the spontaneous process. Initiation of spontaneous reactions is generally performed according to the mechanism plotted in Fig. 3. As compared with the mechanism of chemical conjugation, it shows a significant difference displayed in a principally different role of intermediate compounds in the implementation of one mechanism or another.
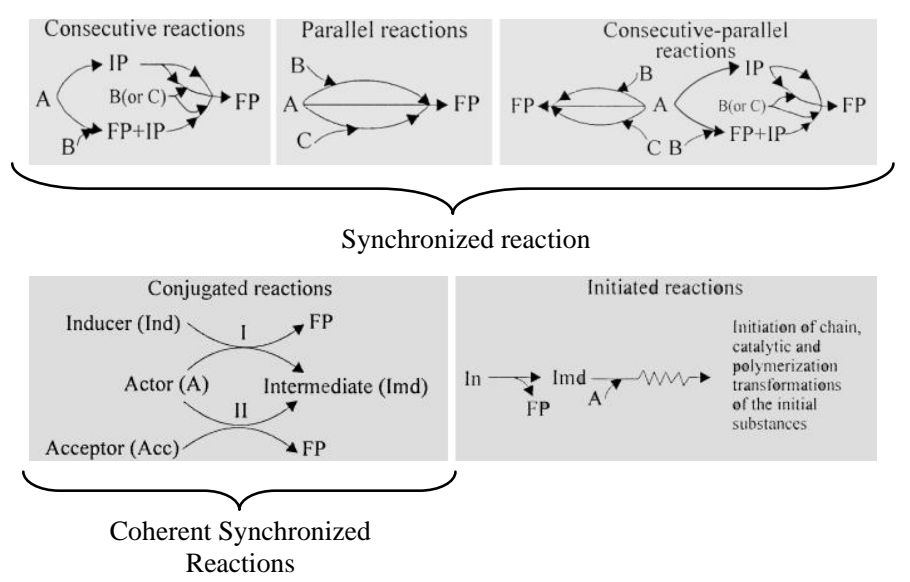

Fig. 3. Schematic layout of interrelated reactions.

Thus, an interaction between reactions may be performed both with the help of stable IP (consecutive reactions), intermediate compounds (initiation, conjugated reactions, etc.), and in their absence (a definite type of parallel reactions). Of special interest for us are interrelated reactions performed with the help of labile, highly reactive intermediate compounds. This question will also be discussed below.

Similar to conjugated (interfering) reactions, parallel reactions must be synchronous, which is their obvious fundamental property.

Meanwhile, consecutive reactions may never be synchronous. This is the principal difference between these two systems - synchronous and usual reactions. As a matter of fact, the final product of the first stage of consecutive reactions is the initial compound for the second stage, therefore, these stages may never be simultaneous (synchronous). Thus, the main difference between synchronous parallel and conjugated (interfering) reactions is that the first type eliminates even a possibility of interaction, whereas in the second case they may only be interacting reactions.

\section{THREE-COMPONENT CONJUGATED PROCESSES}

Let us now discuss different alternatives associated with the participation of the three main components of the conjugated reaction system (actor, inducer and acceptor) in chemical induction and other forms of chemical interference.

One of the common types of actor participation in the induction of the secondary reaction is its transformation to a reactive particle under the inducer effect. This newly formed particle then involves another substance (acceptor) in the secondary reaction. Therefore, the actor participates in gross equations of both conjugated reactions which, finally, are reduced to the widespread type of conjugated reactions (1) and (2).

Let us consider another case in which the actor does not explicitly participate in the gross equation of the secondary reaction. This type of conjugated reactions may be simply represented by the following scheme:

$$
\begin{gathered}
A+I n=F P(\text { final products }) \\
C+A c c=F P
\end{gathered}
$$

Nevertheless, the actor is expended in the secondary reaction, because it participates in the formation of general active, intermediate compound $X$ owing to which communication channels between conjugated reactions are established. It is more desirable to present the induction action mechanism of the primary reaction by the totality of two reactions:

$$
\begin{gathered}
A+I n \rightarrow X \rightarrow F P(\text { primary reaction }) \\
X+C(\text { or } A c c) \rightarrow Y+F P(\text { secondary reaction }) \\
Y+A c c(\text { or } C) \rightarrow X+F P \\
C+A c c=F P(\text { overallreaction })
\end{gathered}
$$

This scheme indicates that $X$ participation in the secondary reaction may be two-fold:

1) $X$ is synthesized at the end of the secondary reaction and, therefore, acts as a catalyst or active site of the chain reaction;

2) $X$ takes part in formation and is exhausted in the secondary reaction, i.e. without regeneration at the end of the secondary reaction.

Then instead of stage (7) the following reaction may be presented:

$$
Y+A c c(\text { or } C)=F P
$$

In the first case, chemical interference is displayed most effectively, because it may cause transformation of high amounts of the substance. Here we consciously use the term 'interference' instead of induction, because in this case (see below) reactions interact not by means of chemical conjugation. The second case is the most typical of conjugated processes. Therefore, it should be discussed in more detail.

Reaction (5) causes an inductive effect resulting in active site (IP) transfer by the reaction (6), which we suggest calling 're-inductive' and then, according to this approach, the general scheme (5) and (6), which takes into account two types of interaction, is transformed to the following scheme of conjugated reactions:

$$
\begin{gathered}
A+I n \rightarrow R I n \rightarrow F P(\text { inductive reaction) } \\
R I n+C \rightarrow Y+F P(\text { re-inductive reaction) } \\
Y+A c c \rightarrow F P
\end{gathered}
$$

Thus, the sequence of reactions (9) and (10) discloses the 
induction effect mechanism of the primary reaction (3) on the secondary reaction (4).

Substance $A$ implicitly participates in the formation of secondary reaction (4) final products, shaped as $Y$, though, of course, a definite amount of $A$ is expended for the formation of other final products, synthesized from $A$ and $I n$ only.

At first sight, a quite paradoxical case is of special interest, when in conjugated reactions the functions of actor and inducer are implemented by the same substance. Such substances may simultaneously act as oxidants or reducer, or possess acid or base properties. This case is demonstrated by the example of hydrogen peroxide (and other peroxides) dissociation, which is commonly described by the following total equation:

$$
2 \mathrm{H}_{2} \mathrm{O}_{2}=2 \mathrm{H}_{2} \mathrm{O}+\mathrm{O}_{2}
$$

when one $\mathrm{H}_{2} \mathrm{O}_{2}$ molecule acts as the oxidant and another molecule as reducer. Active sites, emitted into the system during $\mathrm{H}_{2} \mathrm{O}_{2}$ dissociation, are expended for final product formation in accordance with the target (secondary) reaction [6]. This allows us to accept that one molecule is the actor and another molecule the inducer. In the author's opinion, as two functions are combined in one compound in such a manner, one may state the following about the inductive property of the substance [7]: as transformed to active sites participating in another reaction, an amphoteric substance may be selectively used up in it by a new reaction mechanism.

This type of conjugated reactions may be presented as follows:

$$
\begin{gathered}
A+A(\text { In }) \rightarrow X \rightarrow F P(\text { primary reaction }) \\
A+A c c \rightarrow F P(\text { secondary reaction })
\end{gathered}
$$

Usually, the primary overall reaction (5) consists of elementary stages which lead to $X$ formation and its interaction with the acceptor. The latter interaction may be expressed by the following equation:

$$
X+A c c \rightarrow F P(\text { induced stage) }
$$

As is clear from schemes (11) and (12), only two substances formally participate in such a system of conjugated reactions.

The process by which a complex monomolecular reaction is the primary one may also be related to conjugated reactions:

$$
A \rightarrow X \rightarrow F P \text { (primary reaction) }
$$

In principle, in the alternatives under consideration, the participation of an actor in the secondary reaction, both clearly and implicitly, is permitted.

Thus, conjugated reactions may proceed without the participation of an inducer and it may be valid to assume that substance $A$ possesses the inductive property. In practice, as is commonly known, conjugated processes are processes in which proceeding of one of the reactions induces and speeds up another reaction, not proceeding in the absence of the first one. From these classical positions, there are no arguments in principal against the statement that the primary reaction is monomolecular, e.g. might proceed without inducer.

The example demonstrating this type of conjugation is that of organic peroxide and hydroperoxide decomposition induced by radicals.

The theoretical examples above most encompass the typical features of such systems. These ideas are necessary for relating conjugated processes to one type or another, which would allow specification of the mechanism of particular chemical induction display.

As follows from the last example, inducer participation in conjugated reactions is not obligatory and, therefore, expression

$$
I=\frac{f_{a c c}}{f_{\text {in }}}
$$

where $f_{\text {acc }}$ and $f_{\text {in }}$ are acceptor and inducer consumptions, I - induction factor should be modified so that it becomes suitable for describing all known types of interacting reactions. For this purpose, instead of inducer transformation consideration, it is suggested to take into account the actor expenditure in the denominator for every reaction. Moreover, if the actor does not clearly participate in the chemical equation of the secondary reaction, its expenditure in the form of a highly active intermediate substance must be taken into account in calculations.

\section{The DETERminant EQuATION AND COHERENCE CONDITION OF CHEMICAL INTERFERENCE}

Let us discuss a three-component conjugated reaction:

$$
\begin{gathered}
A+\operatorname{In} \stackrel{w_{1}}{\longrightarrow} X \stackrel{w_{1}}{\longrightarrow} B+\ldots \\
A+A c c \stackrel{w_{3}}{\longrightarrow} C+\ldots
\end{gathered}
$$

To demonstrate, the chemical conjugation mechanism may be presented by the following generalized scheme:

$$
v A+\ln \rightarrow X-\stackrel{1}{\underset{2 a c c}{\longrightarrow} F P} F P
$$

where $v$ is the stoichiometric coefficient.

Comparing this scheme with its common shape expressed by reactions (15) and (16), it should be noted that it gives the best display for the role of the intermediate substance $X$, which is common to both conjugated reactions as the bifurcation factor of the reaction system, and the inducer is expended in amounts providing $A$ expenditure in both reactions.

For the scheme under consideration, the following expression may be deduced:

$$
\frac{1}{v} f_{A}=f_{I n}=\left(f_{A_{1}}+f_{A_{2}}\right) \frac{1}{v}
$$

where $f_{A_{1}}$ and $f_{A_{2}}$ are amounts of actor, used for the synthesis of final products in the primary (1) and the 
secondary (2) reactions, respectively.

Substituting equation (17) into (14), the following expression is deduced:

$$
\begin{aligned}
& D=v\left(\frac{f_{A_{1}}}{f_{A c c}}+\frac{f_{A_{2}}}{f_{A c c}}\right)^{-1} \\
& D=v\left(\frac{w_{A_{1}}}{w_{A c c}}+\frac{w_{A_{2}}}{w_{A c c}}\right)^{-1}
\end{aligned}
$$

where factor $D$ determined in equation (18) is called the determinant.

As follows from equations (17) and (18), it is not obligatory to operate the amount of expended inducer in the calculations. Moreover, there are conjugated processes that proceed without inducer participation. Therefore, it is of much greater importance to account for the expenditure of compound $\mathrm{A}$ in both reactions and to determine the induction factor via it. From these positions, equation (18) is somehow universal, because it reflects chemical induction in any display. Moreover, it is shown below, how many important consequences follow from it, which may not be deduced from equation (14).

Another alternative is also possible, i.e. when in the primary reaction active particles representing catalysts for the secondary reaction are formed. Of course total actor expenditure will then be greater than the inducer expenditure.

Note also that for chemical induction equation (17) is always correct.

Note also an important point: when actor expenditure in conjugated reactions is so high, a large amount of it is used in the primary reaction, when active sites are formed. These sites are IP, and a definite part of them is spent in the formation of final products in the primary and secondary reactions.

It is common knowledge that the primary reaction usually proceeds in several stages (two, at least), and it is of importance that the reactive sites generated in it were mainly expended in the target, secondary reaction. For this purpose, the stages that cause the accumulation of active sites must proceed at a higher rate than the consecutive ones, which give final products of the primary reaction. Let us consider this possibility using the example of conjugated, type (15) and (16a) reactions. It will be shown that relations following from these considerations are also valuable for the specific case of induction-less conjugation of chemical reactions.

When the first stage rate in the primary reaction $\left(w_{1}\right)$ is lower or equals the second stage rate $\left(w_{2}\right)$, the rate of the target product synthesis in the secondary reaction will be defined by $\mathrm{w}_{2}$ and $\mathrm{w}_{3}$ ratio. If $w_{2} \approx w_{3}$, the final products of these conjugated reactions will be accumulated at comparable rates. As $w_{2} \gg w_{3}$, the final products of the primary reaction will mostly be synthesized, which will minimize the induction effect. As $w_{1} \gg w_{2}$ and $w_{2} \gg w_{3}$, the kinetic regularities mentioned are preserved. The only difference is that high concentrations of highly active IP in the reaction system will affect induction efficiency, and the target product synthesis rate will increase somewhat.

The highest induction effect should be expected in the case of inequality $w_{3}>w_{2}$ fulfillment at $w_{1}>w_{2}$. In this regard, accumulation of final products by the overall reaction (16b), No. 2 under the condition of effective induction performance must proceed at an incommensurately higher rate than the primary one. As mentioned above, the induction effect at $w_{2}>w_{3}$ will be insignificant, approaching the limit $I=0$. In this consideration, it is assumed that rate constants $k_{1}, k_{2}$ and $k_{3}$ characterize elementary reactions of the same kinetic order; otherwise, concentration factors would also have to be taken into account which would necessarily complicate the analysis.

It should also be noted that equation (18) gives a more in-depth description of the physical sense of chemical induction phenomenon than equation (14). Thus, by introducing a qualitatively new notion of chemical induction (inducer participation is not obligatory for conjugation proceeding), equation (14) was modified so that it might be used to describe any type of conjugated process. On the other hand, equation (18) is quite universal and may describe other types of interrelated reactions (chemical interference).

Analytical consideration of equation (18) leads to several important consequences for various forms of chemical interference.

1) Consider the case where and $f_{A_{2}} \gg f_{A_{1}}$ and $f_{A c c}$; $\frac{1}{v} f_{A_{1}}=f_{\text {In }}$. From equation (18) it then follows that:

$$
D=\frac{v f_{A c c}}{f_{A_{1}}}
$$

This means that the primary reaction mostly proceeds in the system. Hence, the higher the rate of this reaction, the lower is the inductionfactor $(I)$ which approaches zero in the limit. Obviously, acceptor expenditure is low under these conditions.

2) If $f_{A_{2}} \gg f_{A_{1}}$ threealternatives of the chemical system

with interrelated (interfering) reactions may be realized:

As we neglect the value of the actor part expended for final product synthesis in the primary reaction $\left(f_{A_{1}}\right)$ and under the condition that the actor is completely spent for target product formation only according to schemes (12) and (13), the equation:

$$
f_{A_{2}}=f_{\text {Acc }}
$$

can be deduced. Then equation (18) is reduced to:

$$
D=\frac{v f_{A c c}}{f_{A_{2}}}=v
$$

According to equation (17), the real inducer consumption will equal $A_{2} / v$. In this case, chemical induction is manifested much more effectively. As a consequence, modification of the conjugated reaction system must aim to approach the above-mentioned marginal ratio.

$$
\text { At } f_{A c c} \gg f_{A_{2}} \text { and } \frac{f_{A_{2}}}{v}=f_{I n} \text {, the following expression for }
$$
$D$ is deduced:

$$
D=\frac{v f_{A c c}}{f_{A_{2}}} \gg v
$$

The higher the effectiveness of intermediate substance catalytic action on the secondary reaction or chain transformation of the substrate, the higher is the $D$ value. 
Let us assume that the primary reaction generates an active site in the system, which is the secondary reaction catalyst. It is known that the catalyst does not perform effective work in the reaction, but just speeds it up and remains unchanged at the end of the reaction. At the same time, one of the principles of conjugated reaction principles is that the primary reaction performs effective work for another reaction, conjugated to it via formation of general intermediate substances consumed in both reactions.

Meanwhile, the catalyst may cause or accelerate a spontaneous reaction only and, at most, promote the achievement of outputs close to equilibrium, whereas chemical induction may cause outputs exceeding equilibrium In this regard, if in the primary reaction, active sites representing the catalyst for another synchronously proceeding reaction are accumulated, the latter reaction must be spontaneous and the primary reaction does not perform effective work for its running. On the other hand, in chemical induction, intermediate substances are obligatorily consumed for the secondary, non-spontaneous reaction, chemical energy released in the primary reaction is consumed and, therefore, effective work is performed.

Therefore, catalyst synthesized in the primary reaction is unable to perform effective work in the secondary reaction, and such reactions may not be classified as conjugated ones, because this would entirely contradict the notion of chemical induction.

It is also known from biochemistry that the majority of processes important in biochemistry represent conjugated catalytic reactions. Conjugated catalytic reactions obey the regularities typical of chemical induction, i.e. $D \leq v$, and the catalyst application approaches the system to the ideal shape $(D \approx v)$, and it should be related to the case (A). All these cases are illustrated by the expression (18). It is also shown below how the factor $D$ values help in quantitative assessment of chemical interference effectiveness and determination (under particular conditions) of the type of interaction between reactions dominating in the chemical system studied.

In fact, in the latter case, the catalyst (injected into the system with initial reagents) only speeds up interaction between the IP of the primary reaction and the acceptor. Therefore, $D<v$ and, consequently, chemical conjugation takes place. This means that no matter how the reaction between the acceptor and the IP is intensified by the catalyst, the induction factor (the determinant) may not exceed $v$. When analyzing conjugated catalytic reactions, it should be taken into account that the amount of acceptor involved in the reaction may be significantly increased by application of a catalyst in both conjugated reactions.

Catalyst application to the primary reaction at the stage of IP formation will promote its quick accumulation in the systems in amounts much higher than for its non-catalytic run, and will soften reaction conditions. Secondary reaction is also accelerated. Its proceeding is stipulated by the presence of intermediate substance of the primary reaction.

As $D>>v$, the primary reaction generates in the system a catalyst for secondary, spontaneous reaction. Hence chemical induction is absent. In this regard, a very important conclusion can be drawn: the determinant value of the chemical system characterizes conjugated reactions and represents a common induction factor only in the case where its value fulfills the inequality:

$$
0<D \leq v
$$

Note also that this inequality is typical only of systems in which chemical conjugation occurs. The induction factor correctly describes the three-component system in which owing to the inducer action an IP is synthesized. This product is a reagent but not a catalyst. However, application of the expression (14) shaped equation for the induction factor to other types of interrelated (interfering) chemical reactions leads to wrong values.

Moreover, if the primary reaction is monomolecular, and chemical induction occurs in this system, the induction factor may not be determined from equation (14). This is demonstrated by the limited type of equation (14) application range even to conjugated reactions.

Nevertheless, equation (18) may help in determining the determinant and detecting the type of interrelated reactions from it. It should be noted here that, in the broad sense of the word, interrelated (interfering) reactions are only those proceeding via general intermediate substances, capable reagents, initiators or catalysts of secondary reactions. Otherwise, this class of reaction may be added to by consecutive reactions, which are not coherent.

In fact, for consecutive reactions, the final product of the first stage is the initial product for the following stage and, hence, the reaction mechanism and the reaction rate remain unchanged, and the principle of independent proceeding of separate reactions is also obeyed.

The overwhelming majority of biochemical oxidation processes represent conjugated catalytic (enzymatic) reactions [8]. Therefore, of great importance is the ability to distinguish catalyst and inducer, because any mistake would cause an incorrect interpretation of the chemical mechanisms of the reactions proceeding in the biological system. For instance, redox reaction catalysts are often taken for inducers.

Among interrelated reactions, initiated radical-chain reactions are the most widespread. At the initiation process, the synthesis of highly reactive intermediate compounds (free radicals, in particular) is the necessary condition the target reaction intensification. However, initiating substances are used as additive increments, and the chain initiation stage must proceed at a much lower rate than the chain propagation stage. Otherwise, the chain length becomes shorter, and the chain transformation of the substrate becomes ineffective [9]. In other words, initiators act as 'triggers' of the radical or chain process. From these positions, interacting and initiated reactions are rather similar: catalyst is synthesized in the primary reaction, whereas other reactions generate active sites shaped as free radicals, etc.

In fact, free radicals synthesized in the initiation process may induce spontaneous chain transformation of the acceptor only, which proceeds in the case of quite long chains. Otherwise, the process will not be developed at any noticeable rate.

The determinant of initiated chain reactions will always be higher than one: the longer the chain is, the higher the determinant is. This case is analogous to catalytic influence of the primary reaction on the secondary reaction. Note also that in the case of chemical induction, the primary reaction 
overall accompanies the reaction to be conjugated (which may be non-spontaneous, e.g. induced) and performs effective work for it during its whole run. High amounts of the primary reaction components are taken; hence, the target reaction (if it is of the chain type) may proceed at any chain length, even very short. Thus, chemical induction allows implementation of processes proceeding with extremely short chains, which is absolutely impossible at the initiation.

Sometimes, due to special conditions, chain transformation may hardly be induced. An example of this is the reaction of propyleneepoxidation. However, intense generation of active sites $\left(\mathrm{HO}_{2}\right)$ in the primary reaction gives the possibility of suppressing acceptor chain transformation to undesired products and simultaneously stimulating the main direction - epoxidation. This is obtained due to chemical induction, which induces and speeds up selective transformation of propylene (acceptor) to a quite high rate. The authors have implemented such a conjugation mechanism in propylene epoxidation by hydrogen peroxide [10].

Therefore, free-radical-chain reactions proceeding by short chains (which makes these reactions less profitable for effective transformation into desirable products) may be significantly intensified by chemical induction and, hence, heighten interest in their application.

Assume that $f_{A_{2}}=f_{A c c}$. Then:

$$
D=\frac{v f_{A c c}}{f_{A_{2}}}<v
$$

This means that, in principle, under current conditions chemical induction may take place. However, it is highly improbable that the secondary reaction requiring two actor molecules or more for its run would give a noticeable yield.

Finally, let us consider the case where the actor equals

consumption in both reactions, e.g. $f_{A_{1}}=f_{A_{2}}$. Hence, as with previous examples, several alternatives are possible:

a) $f_{\mathrm{A}_{2}}=f_{A c c}$; then $D=\frac{v f_{A c c}}{\left(f_{A_{1}}+f_{A_{2}}\right)}=\frac{v f_{A c c}}{2 f_{A_{2}}}=\frac{v}{2}$

b) $f_{\mathrm{A}_{2}}>f_{\mathrm{Acc}}$; then $f_{\text {Acc }}<2 f_{\mathrm{A}_{2}}, D<v$

c) $f_{\mathrm{A}_{2}}<f_{\mathrm{Acc}}$; then $\left\{\begin{array}{c}f_{A c c}<2 f_{A_{2}}, D<v \\ f_{A c c}=2 f_{A_{2}}, D=v \\ f_{A c c}>2 f_{\mathrm{A}_{2}}, D>v\end{array}\right.$

We will not give a detailed analysis of case 3, because these alternatives of chemical interference have already been discussed above.

Note that the interference of chemical reactions may alter the effective rate constant of the secondary reaction and break the independence principle of elementary chemical reactions. Moreover, under these conditions non-spontaneous reactions may proceed, which are eliminated in parallel and consecutive reactions.

In case of chemical induction, secondary conjugated reaction may never be of the monomolecular type, because the IP of the primary reaction, being the reagent of the secondary reaction, is consumed in it which naturally leads to a gross equation with more than one component.

The chemical interference range is very broad. It embraces various types of interrelated reactions, including monomolecular secondary (non-conjugated) reactions. The best example is the case in which the primary reaction synthesizes a catalyst for the secondary reaction (which, in principle, may also be monomolecular), and initiates reactions:

$$
\begin{array}{ccccc}
f_{A_{1}} \gg f_{A_{2}} & f_{A_{1}}=f_{A_{2}}=f_{A c c} & f_{A_{2}} \gg f_{A_{1}} ; f_{A_{2}}=f_{A c c} & f_{A_{2}} \gg f_{A_{1}} ; f_{A c c} \gg f_{A_{2}} & f_{A_{2}} \gg f_{A_{1}} ; f_{A c c} \gg f_{A_{2}} \\
D=0 & D=v / 2 & D=v & D>v & D \gg v
\end{array}
$$

Chemical interference determinant scale; $D=0 \div v$ is the chemical conjugation range $D>v$ is the range of other interactions proceeding

The necessary condition for chemical interference realization in the reaction system is the spontaneous type of its primary reaction.

Based on the data presented in this chapter, the above equation shows the determinant scale, which makes the detection of one type of reciprocal influence of chemical reactions (chemical interference) or another in the reaction mixture easier.

Kinetic studies of the chemical system with the interaction between reactions, based on the experimental data, allow selection between various types of interfering chemical reactions. Therefore, chemical interference investigations may be found useful for the study of reaction mechanisms.

It seems to the authors that one of the forms of self-organization in complex chemical systems may be chemical interference, described by the determinant equation (18). This idea is also confirmed by the fact that the overwhelming majority of enzymatic reactions in vivo are synchronized and conjugated. As shown below, the determinant equation is quite useful and easily adjustable for solving complex chemical tasks.

Physicochemical features of chemical interference are displayed by theoretical kinetic curves in Fig. 4 and allow the discovery of some shapes, which will be discussed below.

These idealized curves were composed with the hypothesis that primary reaction (1) in the absence of secondary reaction (2) proceeds until the end, i.e. its initial reagents are consumed completely. Therefore, acceleration of the secondary reaction is studied under conditions in which the primary reaction runs completely to its end.

The curves in Fig. 4(a) show that in the absence of the secondary reaction (2) the primary reaction (1) proceeds with almost $100 \%$ consumption of the reagents. As secondary reaction (2) products are accumulated, the quantity of primary reaction products decreases and both curves pass via extreme points (peaks). According to theoretical notions, the maximum of reaction (2) corresponds to the minimum of 
reaction (1). In the case where, due to kinetic reasons the reaction (2) is synchronized with the primary reaction (1) with some delay, a phase shift $(\Delta)$ occurs, shown by a dashed line in Fig. 4(a). The maximum on this curve is right shifted by $\Delta$ value. In other words, the phase shift means the difference between the primary reaction minimum and the secondary reaction maximum.

Based on kinetic regularities following from the type of these curves, one may make an important conclusion that for every particular condition, the totality of reaction products will correspond to a constant value of the actor consumption or, inaccordance with stoichiometry (scheme 3 ) of the inducer and the assumption (e.g. postulation) of its complete consumption, the following expression (coherent correlation) becomes valid:

Coherent Correlation

$$
\frac{1}{v} f_{A}=f_{I n}=f_{A_{1}}+f_{A_{2}}=f_{A_{1}}^{\prime}+f_{A_{2}}^{\prime}=f_{A_{1}}^{\prime \prime}+f_{A_{2}}^{\prime \prime}=\ldots=\text { const }
$$

It is assumed that equation (19) is the coherence condition for chemical interference, at least for the case in which the $D$ value varies between zero and $v$, i.e. chemical conjugation takes place.

An important consequence of equation (19) should be outlined: effective interference between two chemical reactions is observed in the case where, in the absence of the secondary reaction, the primary reaction proceeds completely to its end in the whole range of conditions.
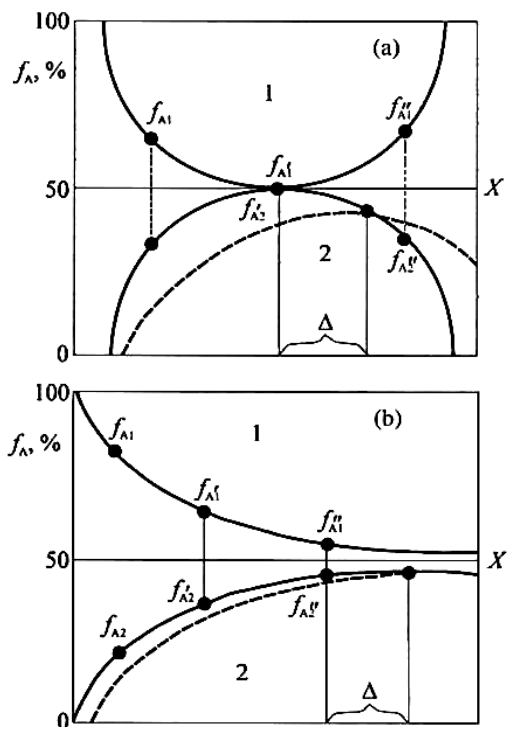

Fig. 4. Theoretical kinetic curves for interfering reactions (primary 1and secondary 2) of extreme (a) and asymptotic (b) type; $\Delta$ is the phase shift.

Another case, also shown in Fig. 4(b), is characterized by curves free from extreme points, approaching the $X$ level. Such curve shapes indicate zero concentration of the actor and general highly reactive intermediate particles in the area where asymptotic curves approach the $X$ level most closely. Therefore, at asymptotic approach no products are formed by interfering reactions. The coherence condition, displayed by equation (19), is also fulfilled in this case.Other cases are mostly associated with incomplete (i.e. partial) proceeding of the primary reaction in the absence of the secondary reaction and its complication by side reactions, especially with participation of an inducer. In the cases under consideration, as actor or inducer consumptions per primary and secondary reactions are calculated, the amounts consumed for the synthesis of side products must be subtracted from the total amount or, at incomplete consumption, the amounts of non-reacted $A$ or In compounds.

Note that the $X$ line in Fig. 4(b) may be located above or below the $50 \%$ level of product accumulation from both interfering reactions or actor (inducer) and acceptor consumptions. Line location above the $\mathrm{X}$ line means that the greater part of the total, highly active intermediate particles (active sites) is consumed for secondary reaction product formation and, vice versa, when the line is below $X$ level.

A dashed curve in Fig. 4(b) shows the phase shift, the origin of which is similar to that in Fig. 4(a).

It is known that the correspondence principle suggested by N. Bohr (1923) postulates that 'any theory pretending for better description and broader application range than the older one must include the latter in the form of marginal case'.

The succession of theoretical notions may be shown by using the corresponding principle for consideration of the chemical interference theory as a more general concept of interrelated and interacting reactions. The correspondence principle applied to the interference of chemical reactions must represent a postulate, which in the marginal case of the determinant $(D \rightarrow v)$ requires the coincidence of its chemical consequences with yields of usual chemical reactions, e.g. classical stoichiometric reaction.

For conjugated reactions, the maximum high determinant value equals $v(D=v)$. This means that only a secondary reaction proceeds in the system, coinciding by stoichiometric parameters with the corresponded conjugated reaction. Simultaneously, the level $D=v$ is the lowest for initiation, autocatalysis, chain and other types of interfering reactions which, in the previous case, are reduced to a stoichiometric reaction. Hence, when considering the suggested theory from the position of the correspondence principle, the following conclusion can be made: under the condition $D=v$, the conjugated, chain, initiated and autocatalytic reactions are necessarily reduced to stoichiometric reactions, i.e. described in the framework of the classical theory of chemical reactions. Formally, transition to a stoichiometric reaction happens at $D$ $\rightarrow v$, which emphasizes the general type of the new notion of interfering chemical reactions.

\section{INTERFERENCE OF HYDROGEN PEROXIDE DISSOCIATION AND SUBSTRATE OXIDATION REACTIONS}

The examples given below, for instance, methane oxidation to methanol and propylene oxidation to propylene oxide, demonstrate experimental approaches to the study of interfering reaction dynamics and, with the help of the determinant equation, the potential abilities of reaction media are assessed and the type of chemical interference determined.

Monooxigenase reaction for synthesizing methanol from methane was studied in the presence of cytochrome P-450 biosimulators, such as ferroprotoporphyrin catalysts with the 
carriers $\left(\mathrm{A}_{2} \mathrm{O}_{3}, \mathrm{NaX}\right.$, aluminum-chromium-silicate and aluminum-magnesium- silicate). This reaction helped in the detection of the highest catalytic activity for $\mathrm{PPF}^{3+} \mathrm{OH} /$ aluminum-magnesium-silicate [11], which also displayed the highest catalytic activity for hydroxylation reaction. As shown, optimal hydroxylic activity of the catalyst is displayed in the initial $30 \mathrm{~min}$ of its operation (methanol output equals $60 w t \%$, selectivity is $97 w t \%$ ). Fig. 5 shows that kinetic dependence of methanol output on temperature has a maximumat $180^{\circ} \mathrm{C}$, and the curve of molecular oxygen yield has aminimum. In this experiment, methanol yield reaches 46.5 wt.\%, which at methane conversion rises to 48wt.\%. Nontarget products $\mathrm{CH}_{2} \mathrm{O}$ and $\mathrm{HCOOH}$ in low amounts $(\approx 1.5 \%)$ and temperature cause no effect on their yield. Comparison of the curves 2 and 5 in Fig. 5 in the framework of the ideas discussed above shows their reliable analogy with the theoretical curves in Fig. 4(a). Some deviation of coherence $\left(f_{\text {Ind }}\right)$ from the theoretical level may be explained by synthesis of side oxidation products and systematic errors, which usually accompany any chemical experiment. However, its value $\left(f_{\text {Ind }}\right)$ obeys the main coherence condition following from equation (19). More precisely, $f_{\text {Ind }} \approx$ constant for current reaction conditions. This value may be simply calculated from the data of Fig. 5(a). A graphic presentation of chemical interference, shaped as asymptotically approaching curves in another range of the reaction conditions, is plotted in Fig. 5(b). Comparison of the experimental curves from Fig. 3(b) with the theoretical ones from Fig. 4(b) indicates their adequacy and relates the observable chemical interference to the case above $X$, i.e. when the $\mathrm{CH}_{4}$ oxidation rate slightly exceeds the rate of molecular oxygen synthesis.
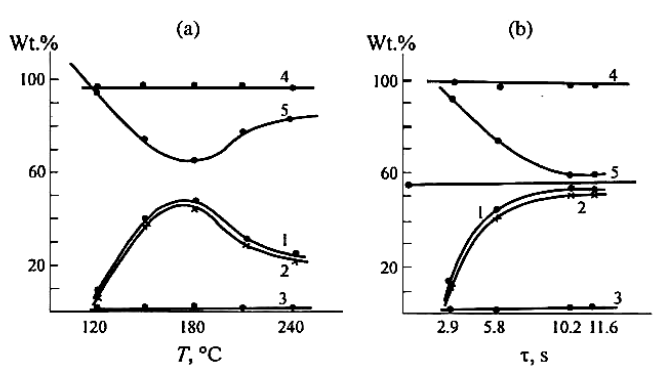

1) $\mathrm{CH}_{4}$ conversion; 2) $\mathrm{CH}_{3} \mathrm{OH}$ output; 3) $\mathrm{CH}_{2} \mathrm{O}$ and $\mathrm{HCOOH}$ outputs; 4) selectivity; 5: $\mathrm{O}_{2}$ output Ratios: $\mathrm{CH}_{4}: \mathrm{H}_{2} \mathrm{O}_{2}=1: 1.4$ (a) and 1:1.8 (b); $V_{\mathrm{CH}_{4}} V_{\mathrm{H}_{2} \mathrm{O}_{2}}=0.8 \mathrm{ml} / \mathrm{h},\left[\mathrm{H}_{2} \mathrm{O}_{2}\right]=20 w t \%$.

Fig. 5. Dependencies of methane hydroxylation outputs on (a) temperature and (b) contact time at $180^{\circ} \mathrm{C}$.

In the chemical system studied biosimulator catalyzes two interrelated (catalase and monooxygenase) reactions, which are synchronized and proceed according to the following mechanisms (see Fig. 6).

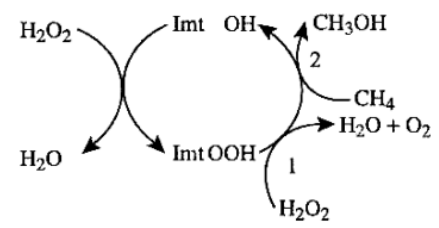

Fig. 6. The mechanism of the coherent-synchronized catalase and monooxigenase $\left(\mathrm{CH}_{4}+\mathrm{H}_{2} \mathrm{O}_{2}=\mathrm{CHOH}+\mathrm{H}_{2} \mathrm{O}\right)$ reactions. where $\mathrm{ImtOH}$ is $\mathrm{PPFe}{ }^{3+} \mathrm{OH} / \mathrm{AlMgSibiosimulator;} \mathrm{ImtOOH}$ is $\mathrm{PPFe}^{3+} \mathrm{OH} / \mathrm{AlMgSi}$ intermediating compound: (1) primary catalase reaction and (2) hydroxylation (secondary monooxygenase reaction).

Both reactions (1) and (2) in the scheme proceed via general $\mathrm{PPFe}^{3+} \mathrm{OH} / \mathrm{AlMgSi}$ intermediating compound, which certainly is the transferring agent for the inductive action of the primary reaction to the secondary reaction. The determinant calculated by equation (18), which allows quantitative identification of an interaction between reactions, equals:

$$
D=0.48
$$

This indicates that reactions (1) and (2) are conjugated, because the value obtained on the determinant scale of chemical interaction (Fig. 3) falls within the range of chemical conjugation $(D<1)$, because in the current case $v=1$, (see Fig. 7).

The diagrams in Fig. 4 illustrate the conjugated type of two reactions: $\mathrm{H}_{2} \mathrm{O}_{2}$ dissociation and propylene epoxidation by hydrogen peroxide [12]. Actually, the rate decrease of biosimulator catalase activity product $\left(\mathrm{O}_{2}\right)$ accumulation is accompanied by the rate increase of epoxidation product synthesis, and these processes interfere via general highly active intermediating compound: per-FTPhFe ${ }^{3+} \mathrm{OOH} / \mathrm{Al}_{2} \mathrm{O}_{3}$.

However, presenting the interference picture via a diagram has several principal disadvantages: 1) Diagrams do not show how coherence is implemented; 2) Phase shifts may not be shown; 3) maxima and minima in accumulation of products of both reactions and 4) the absence of asymptotic curves.

The advantage of the diagrams is that they are highly illustrative of chemical conjugation between current reactions. Thus, diagrams help in demonstrating one of the aspects of chemical interference associated with conjugation of the processes.

Let us consider the experimental data shown in Fig. 8(a) and Fig. 8(b), obtained at homogeneous gas-phase oxidation of methane (or natural gas) by hydrogen peroxide to methanol under pressure [13], [14]. The increase in contact time to $0.95 \mathrm{~s}$ (Fig. 8(a)) gives a maximum of methanol output and a minimum of oxygen output. A further increase in the contact time reduces methanol output, whereas molecular oxygen output increases. A similar kinetic regularity is observed in experiments with variable pressure (Fig. 8(b)).

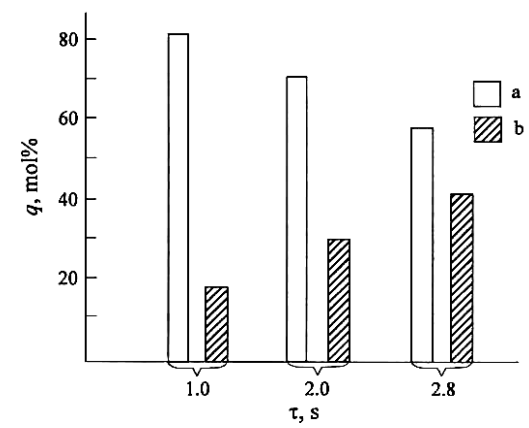

Fig. 7. Hydrogen peroxide consumption $(q)$ in catalase (a) and monooxygenase (b) reactions with time of contact; $T=200^{\circ} \mathrm{C}, \mathrm{C}_{3} \mathrm{H}_{6}: \mathrm{H}_{2} \mathrm{O}_{2}=$ $1: 1.2(\mathrm{~mol})$.

Thus, comparison of the curves of molecular oxygen accumulation and $\mathrm{CH}_{4}$ consumption (or $\mathrm{CH}_{3} \mathrm{OH}$ 
accumulation) shows that the maximum of $\mathrm{CH}_{4}$ transformation corresponds to the minimum of $\mathrm{O}_{2}$ accumulation.

Chemical interference is clearly displayed owing to almost $100 \%$ selectivity of reactions: increased $\mathrm{O}_{2}$ synthesis induces a simultaneous decrease of $\mathrm{CH}_{4}$ transformation to $\mathrm{CH}_{3} \mathrm{OH}$ and vice versa.

As the curves in Fig. 8(a) and (b) are considered from positions of coherence and possible phase shift, note that the particular reaction mixture differs from the mixtures considered above by relatively low (about 20wt.\%) $\mathrm{CH}_{4}$ substrate conversion, although $\mathrm{H}_{2} \mathrm{O}_{2}$ dissociates almost completely. This circumstance must be taken into account in the framework of the approach to such a case described above.

Experimental detection of chemical interference determinant by the following equation:

$$
D=v\left(\frac{r_{1}}{r_{\mathrm{CH}_{4}}}+\frac{r_{2}}{r_{C H_{4}}}\right)^{-1}
$$

where $r_{1}, r_{2}$ and $r_{\mathrm{CH}_{4}}$ are actor $\left(\mathrm{H}_{2} \mathrm{O}_{2}\right)$, inducer $\left(\mathrm{H}_{2} \mathrm{O}_{2}\right)$ and acceptor $\left(\mathrm{CH}_{4}\right)$ consumption rates, respectively; $v$ is the stoichiometric coefficient equal to 1 for the current conditions of minimal $\mathrm{O}_{2}$ and maximal $\mathrm{CH}_{3} \mathrm{OH}$ outputs, gave $D=0.18$. On the chemical interference scale in Fig. 1, this value falls within the range for conjugated reactions. It quantitatively characterizes the inductive action of $\mathrm{H}_{2} \mathrm{O}_{2}$ on $\mathrm{CH}_{4}$ oxidation and indicates the presence of high potential abilities to increase the induction effect of the system studied (theoretically, in the current case, $D$ may increase to 1 or will tend to approach at least the $50 \%$ level) [2]. There are physicochemical experimental techniques that allow manipulation of conjugating reaction rates. On the other hand, not applying the method of stationary concentrations, the determinant equation (18) gives an opportunity of analyzing the kinetics of complex reactions with insignificantly studied mechanisms. For these two reactions the conjugation mechanism is:

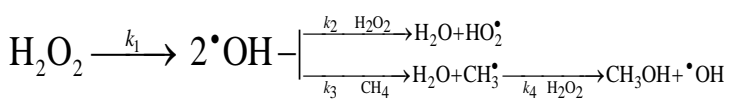

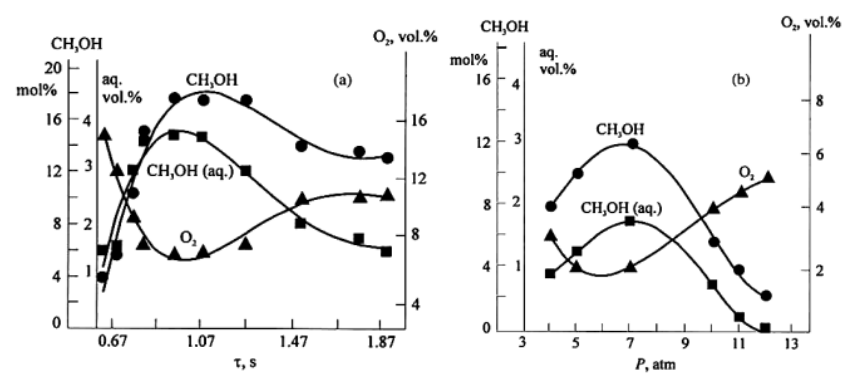

Fig. 8. The dependence of methanol output on the contact time (a) and pressure (b); $\mathrm{T}=400^{\circ} \mathrm{C},\left[\mathrm{H}_{2} \mathrm{O}_{2}\right]=30 \mathrm{wt} \%$ (a) $\mathrm{p}=7 \mathrm{~atm} ; V_{\mathrm{CH}_{4}}=31.4 \mathrm{l} / \mathrm{h}$;

$V_{\mathrm{H}_{2} \mathrm{O}_{2}}=0.18 \mathrm{l} / \mathrm{h} ; \mathrm{CH}_{4}: \mathrm{H}_{2} \mathrm{O}_{2}=1: 1.4(\mathrm{~mol})$ and (b) $V_{\mathrm{H}_{2} \mathrm{O}_{2}}=0.18 \mathrm{1} / \mathrm{h} ; V_{\mathrm{CH}_{4}}=$ $62.41 / \mathrm{h} ; \mathrm{CH}_{4}: \mathrm{H}_{2} \mathrm{O}_{2}=1: 0.4(\mathrm{~mol})$.

As follows from the determinant equation:

$$
r_{A c c}\left(\mathrm{CH}_{4}\right)=\frac{D}{v}\left(r_{A_{1}}+r_{A_{2}}\right)
$$

or

$$
r_{\mathrm{CH}_{4}}=\frac{D}{v}\left(k_{2}\left[\mathrm{H}_{2} \mathrm{O}_{2}\right]+k_{3}\left[\mathrm{CH}_{4}\right]\right)[\mathrm{OH}]
$$

Using experimentally obtained values of $r_{\mathrm{CH}_{4}}$ and $D$ [8], the appropriate kinetic calculations were carried out. Therefore, equation (22) adequately describes the kinetics of interfering reaction (21).

Thus, the determinant equation was found useful for the analysis of the kinetics of complex reactions in that it made simpler the kinetic calculations at determination of the kinetic model of interrelated and synchronized reactions proceeding in the reaction mixture and also the qualitative and quantitative assessment of chemical interference itself.

Note also that some authors [11]-[14] have had to use all their inventiveness in order to impart high experimental demonstrativeness to chemical interference.

\section{THE DIFFERENCE BETWEEN INITIATION AND INDUCTION PHENOMENA}

Since no clear distinction between initiation and induction of chemical reactions is made in the scientific literature, and very often one event is taken for another, we will discuss this question in more detail.

Active sites are generated both in initiation reactions and by chemical induction, and this situation is common to all types of initiated and conjugated reactions. The main difference is related to the effect of these active sites on the target reaction (in the case of chemical induction, this is the secondary reaction). Only one aspect - generation of active sites - is very often considered. On this basis many authors make their own conclusions.

Meanwhile, only the determination of further behavior of active particles in the system - the influence on the target reaction - may exactly determine particular cases of initiation and induction.

By way of example, let us consider how radical polymerization of vinyl monomers is initiated by free hydroxyl radicals. These radicals are obtained by catalytic action of bivalent iron salts on $\mathrm{H}_{2} \mathrm{O}_{2}$ dissociation (Fenton reagent) [15]:

$$
\begin{aligned}
& \mathrm{Fe}^{2+}+\mathrm{H}_{2} \mathrm{O}_{2} \rightarrow \mathrm{Fe}^{3+}+\mathrm{OH}^{-}+{ }^{\bullet} \mathrm{OH} \text { (initiation) } \\
& \cdot \mathrm{OH}+\mathrm{CH}_{2}=\mathrm{CHCN} \rightarrow \mathrm{HOCH}_{2} \dot{\mathrm{C}} \mathrm{HCN} \stackrel{\mathrm{CH}_{2}=\mathrm{CHCN}}{\longrightarrow} \\
& \rightarrow \mathrm{HO}\left(\mathrm{CH}_{2}=\mathrm{CHCN}\right)_{n} \mathrm{CH}_{2} \dot{\mathrm{C}} \mathrm{HCN} \text { (chain propagation) }
\end{aligned}
$$

Every free ${ }^{\circ} \mathrm{OH}$ radical initiates one reaction chain. After the addition of a single monomer molecule it rests at the inactive chain end and does not participate in subsequent propagation acts. A single ${ }^{\circ} \mathrm{OH}$ radical induces transformation of a high amount of monomer and $\mathrm{H}_{2} \mathrm{O}_{2}$ acts as the initiator, but does not participate in the gross equation of the target reaction. As suggested by Dolgoplosk and Tinyakova, $\mathrm{H}_{2} \mathrm{O}_{2}$ excess over $\left.\mathrm{Fe}^{2+}\left(\left[\mathrm{H}_{2} \mathrm{O}_{2}\right] / \mathrm{Fe}^{2+}\right]>1\right)$ inhibits polymerization [16], [17].

There are other examples characterizing the initiating action of $\mathrm{H}_{2} \mathrm{O}_{2}$. Ashmore [15] indicates that primary radicals 
do not usually induce chain degradation, because they are captured by a monomer molecule before a sufficient number of collisions with diluted initiator particles.

Therefore, $\mathrm{H}_{2} \mathrm{O}_{2}$ concentration in the system, where it acts as the initiator, is strictly limited. Initiators are applied as highly diluted solutions and accelerate only reactions, which proceed at a high rate after initiation. The initiator concentration increase in the system is limited also for chain reactions involving short chains.

This restriction for initiation reactions was set by Benson: as the chain propagation rate is 10-100 times higher than the initiation rate of active sites, propagation reactions dominate in the system and the chain process is of great importance [9]. Here, the following condition must be fulfilled for initiated chain reactions:

$$
v=\frac{w_{\text {prod }}}{w_{\text {ini }}}>10
$$

where $v$ is the chain length.

From this expression it is clear that initiation is valuable for chain lengths, at least, above 10 units, i.e. the chain propagation rate must be at least 10 times above the initiation rate.

According to data by Dolgoplosk and Tinyakova [17], an increase in $\mathrm{H}_{2} \mathrm{O}_{2}$ concentration inhibits the polymerization process, initiated by low concentrations of this compound. This gives rise to the question: What is the further behavior of active sites? Ashmore suggests that in the presence of other organic substances the redox system may oxidize active sites. For example, the 'ferrous iron-hydrogen peroxide' easily oxidizes aromatic compounds [15]. Thus, an interesting interrelation between polymerization and oxidation reaction and the possibility of transferring from one reaction to another with $\mathrm{H}_{2} \mathrm{O}_{2}$ concentration change is observed.

One more example of the conjugated process is illustrated by benzene oxidation reaction in the Fenton system, which is discussed in detail in the book by Emanuel and Knorre [18]. Aqueous benzene solution does not directly interact with $\mathrm{H}_{2} \mathrm{O}_{2}$. However, as bivalent iron salt is added to the system, $\mathrm{Fe}^{2+}$ ion oxidation to $\mathrm{Fe}^{3+}$ is accompanied by benzene oxidation to phenol and diphenyl. Therefore, under these conditions $\mathrm{H}_{2} \mathrm{O}_{2}$ may not oxidize benzene. A successful oxidation performance requires $\mathrm{H}_{2} \mathrm{O}_{2}$ dissociation to hydroxyl radicals with $\mathrm{Fe}^{2+}$ ions as catalysts. Then free ${ }^{\circ} \mathrm{OH}$ radicals react with benzene inducing its oxidation. Therefore, these two reactions $-\mathrm{H}_{2} \mathrm{O}_{2}$ dissociation induced by $\mathrm{Fe}^{2+}$ catalyst (primary reaction) and benzene oxidation by hydrogen peroxide (secondary reaction) - are conjugated. In this case, $\cdot \mathrm{OH}$ radicals are carriers of $\mathrm{H}_{2} \mathrm{O}_{2}$ inductive action on the secondary reaction.

As follows from this example, catalytic dissociation of $\mathrm{H}_{2} \mathrm{O}_{2}$ generates ${ }^{\circ} \mathrm{OH}$ radicals in the system and under definite conditions induces another reaction. In this regard, with respect to the particular objective of generating free radicals to the system, $\mathrm{H}_{2} \mathrm{O}_{2}$ may possess properties of initiator (of polymerization, e.g.) and inducer (of benzene oxidation by hydrogen peroxide, e.g.).

There is a special case in which both conjugated reactions proceed at the maximal rate and, hence, chemical interference is observed in a specific manner, which is realized in the membrane catalysis of mitochondrial energetic process.

\section{CONCLUSION}

The strategy associated with imparting high efficiency and orderliness to chemical interference has proved itself:

1) The primary reaction runs with almost $100 \%$ conversion in the absence of the secondary reaction;

2) It approaches $100 \%$ selectivity for both reactions.

The study of chemical interference and its particular case of conjugated processes indicate that it may represent a simple prototype for similar systems realizable in biochemical systems.

First of all, realization of chemical interference is associated with the selection of those reactions that are capable of self-organization, i.e. to formation of complex reaction ensemble. The ensemble of molecules and, as a consequence, the ensemble of reactions is able to interfere, because aggregation of molecules in ensembles somehow creates an algorithm for the realization of mutually agreed spontaneous reactions. Contrary to free molecules, the distinctive feature of an ensemble of molecules is the fact that structural organization of an ensemble of molecules allows the running of both simple and complex reactions, chemical interference of which is vitally important for the living system activity. In this discussion we would like to indicate that chemical interference is the necessary property of biochemical systems. Note also that molecular ensembles may be differently organized structurally and, therefore, the type of ensemble from the same molecules is responsible for proceeding of one type of interrelated reactions or another (i.e. chemically interfering reactions).

The ensemble of reactions is self-organized through the intermediary of general highly active substances. These processes may be accelerated and effectively implemented with the help of catalysts similar to processes, which take part in the living systems.

Thus, self-organization of an ensemble of reactions capable of being intensified or weakened and, therefore, inducing chemical interference, may be suggested as the basis for the principle of organization of many enzymatic ensembles.

Of great interest is the creation of trigger reaction ensembles, which will not only change the interference picture but also the type of interacting reaction with respect to the action of temperature, pressure, medium $\mathrm{pH}$ and other important factors.

\section{REFERENCES}

[1] T. M. Nagiev, Coherent Synchronized Oxidation by Hydrogen Peroxide, Elsevier, Amsterdam, 2007, p. 325.

[2] T. M. Nagiev, Zh. Fiz. Khim., 1994, vol. 68, no. 3, pp. 456-460.

[3] T. M. Nagiev, Zh. Fiz. Khim., 2000, vol. 74, no. 11, pp. 2034-2040.

[4] A. L. Buchachenko, Usp. Khim., 1999, vol. 68, no. 2, pp. 99-118.

[5] G. K. Boreskov, Catalysis, Pt. 1/2, Nauka, Novosibirsk, 1971, p. 267

[6] T. M. Nagiev, Khim. Fiz., 1983, no. 6, pp. 823-832.

[7] D. Nonhibel and J. Walton, Chemistry of Free Radicals, Mir, Moscow, 1977, pp. 606.

[8] B. Nulman and A. Pulman, Quantum Biochemistry, Mir, Moscow, 1965, pp. 654

[9] S. Benson, Thermochemical Kinetics, Mir, Moscow, 1971, p. 308. 
[10] T. M. Nagiev, G. M. Mamed'yurov, Z. M. Nagieva, and S. I. Agaeva, XII Mendeleev Council on General and Applied Chemistry, Thes. Rep. Inform., Nauka, Moscow, 1981, no. 4, p. 268.

[11] T. M. Nagiev and M. T. Abbasova, Zh. Fiz. Khim., 1997, vol. 71, no. 7, pp. 1213-1217.

[12] T. M. Nagiev, L. M. Galanova, S. Z. Zulfugarova, and C. A. Mustafaeva, Zh. Fiz. Khim., 1996, vol. 70, no. 11, pp. 2063-2068.

[13] E. M. Mamedov, E. G. Farajev, and T. M. Nagiev, Azerb. Khim. Zh., 1997, no. 1-4, pp. 45-48.

[14] T. M. Nagiev, E. G. Farajev, and E. M. Mamedov, Zh. Fiz. Khim., 2001, vol. 75 , no. 1 , pp. $50-56$

[15] P. A. Ashmore, Catalysis and Inhibition of Chemical Reactions, Mir, Moscow, 1966, p. 386.

[16] B. A. Dolgoplosk and E. I. Tinyakova, Generation of Free Radicals and Their Reactions, Nauka, Moscow, 1982, p. 253.

[17] B. A. Dolgoplosk and E. I. Tinyakova, Redox System as the Source of Free Radicals, Nauka, Moscow, 1972, p. 237.

[18] N. M. Emanuel and D. G. Knorre, The Course of Chemical Kinetics, Vysshaya Shkola, Moscow, 1984, p. 237.

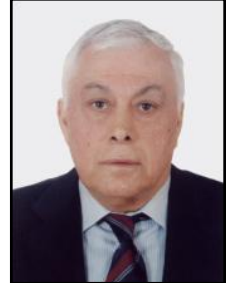

Tofik M. Nagiev was born in Baku Azerbaijan, on March 4, 1941. He was graduated from the Chemistry Faculty of Baku State University. He is a philosophy doctor on physical chemistry field in Institute of Catalysis and Inorganic Chemistry Baku, Azerbaijan, since 1967 and obtained his doctor of science degree on petrochemical and petrochemical synthesis field in Institute of Petrochemical Synthesis named after A. V. Topchiyev Moscow, Russia, in 1973

$\mathrm{He}$ is the vice-president of Azerbaijan National Academy of Sciences, the director of Research Center of "Azerbaijan National Encyclopedia" and the department chief of Institute of Catalysis and Inorganic Chemistry named after Acad. M. F. Nagiev of ANAS. He is also a professor of the Department of the Physical and Colloid Chemistry of Baku State University.

He is a member of New York Academy of Sciences, a foreign member of the scientific council on catalysis of the Department "Chemical Science and Materials" of Russian Academy of Sciences. 